\title{
A Systematic Review on the Psychological Effects of Perfectionism and Accompanying Treatment
}

\author{
Hahna Patterson1,2, Casey Mace Firebaugh ${ }^{1,3 *}$, Tara Rava Zolnikovi,4, Rebecca Wardlow ${ }^{1}$, \\ Stephanie M. Morgan ${ }^{1}$, Brett Gordon ${ }^{1,5,6}$
}

\author{
${ }^{1}$ School of Behavioral Sciences, California Southern University, Costa Mesa, California, USA \\ ${ }^{2}$ University of New England, Portland Maine, USA \\ ${ }^{3}$ Department of Health Sciences, Central Washington University, Ellensburg, Washington, USA \\ ${ }^{4}$ Department of Community Health, National University, La Jolla, California, USA \\ ${ }^{5}$ College of Doctoral Studies, University of Phoenix, Phoenix, Arizona, USA \\ ${ }^{6}$ College of Business, Embry-Riddle Aeronautical University, Daytona Beach, Florida, USA \\ Email:^macec@cwu.edu
}

\begin{abstract}
How to cite this paper: Patterson, $\mathrm{H}$., Firebaugh, C. M., Zolnikov, T. R., Wardlow, R., Morgan, S. M., \& Gordon, B. (2021). A Systematic Review on the Psychological Effects of Perfectionism and Accompanying Treatment. Psychology, 12, $1-24$.

https://doi.org/10.4236/psych.2021.121001
\end{abstract}

Received: November 17, 2020

Accepted: January 5, 2021

Published: January 8, 2021

Copyright $\odot 2021$ by author(s) and Scientific Research Publishing Inc. This work is licensed under the Creative Commons Attribution International License (CC BY 4.0).

http://creativecommons.org/licenses/by/4.0/

\begin{abstract}
Maladaptive perfectionism can have an injurious and consequential impact on individuals and society with effects that may contribute negatively to a wide range of mental and physical health issues. Not only does perfectionism make people more susceptible to physical and mental health disorders, but its perniciousness also makes the inevitabilities of hardship and illness more challenging to cope with when they do occur. The purpose of this review was to investigate the psychological and physical impacts of maladaptive perfectionism on individuals and best practices for accompanying treatment. A systematic review was performed to search, review, analyze, and synthesize the existing literature. Suggestions for practical treatment recommendations and potential areas of further inquiry are offered. PRISMA checklist was utilized for inclusion in the review and 118 articles met search criteria. The broader emerging themes were: etiology and progression, psychological consequences of perfectionism, and available treatments. Concerns related to the multitude of downstream psychological impacts are highlighted, including the emerging evidence regarding perfectionism as both a primary and secondary disorder along with current and emerging interventions.
\end{abstract}

\section{Keywords}

Perfectionism, Maladaptive Perfectionism, Cognitive Behavioral Therapy, Self-Efficacy, Social Cognitive Theory, Psychodynamic Therapy 


\section{Introduction}

Perfectionism is broadly conceptualized as a personality style associated with a drive to achieve beyond a reasonable standard and accompanied by marked feelings of self-criticism (Frost et al., 1990). Perfectionism is pervasive and increasing in Western culture, affecting various age groups, ethnicities, and socio-economic classes (Burgess \& Dibartolo, 2016). A meta-analysis conducted by Curran and Hill (2019), found perfectionism has increased 32\% in the birth cohort of United States, Canadian, and British students from 1989-2016. While perfectionism impacts cognition, emotion, and behavior in individuals across the lifespan, it is implicated in increased mortality rates amongst older adults and Fry and Debats (2009) reported a 51\% increase in the risk of death for those who are high in self-expectations versus those who score low on scales of perfectionism. The deleterious effects of maladaptive perfectionism are manifold and associated with significant psychological and physical effects negatively impacting multiple domains of an individual's life.

\subsection{Background of the Problem}

The roots of the scientific study of perfectionism can be traced to psychoanalytic theory and in particular, the work of Karen Horney and Alfred Adler (Flett \& Hewitt, 2019; Flett et al., 2017; Smith et al., 2018). Psychoanalytic treatment focused on the notion that perfectionism has roots in neuroticism and resulting behaviors were a form of over-compensation (Smith et al., 2018). Scant attention was placed on conceptualizing perfectionism until the 1990's when perfectionism was defined and operationalized with a set of theoretical underpinnings, promoting increased interest in the construct and driving research in the area (Frost et al., 1990; Flett et al., 1992).

Hewitt and Flett (1991) developed a prominent and well-studied paradigm, the multidimensional theory of perfectionism (De Cuyper et al., 2015; Stoeber, 2018). The model suggests that perfectionism consists of three interrelated components: self-oriented, other-oriented, and socially prescribed thinking styles. Self-oriented perfectionism is the propensity for individuals to set and be driven by self-imposed high standards. Other-oriented perfectionism is the proclivity toward holding others to standards that are not realistic. Socially prescribed perfectionism is the belief that others hold them to standards that cannot be reasonable and consistently met (Hewitt \& Flett, 1991).

Perfectionism is often classified as either adaptive or maladaptive. If adaptive, it provides motivation to set and achieve goals and, as a result, yields a variety of positive emotions and overt measures of success (Akay \& Bratton, 2017; Hewitt \& Flett, 1991; Lo \& Abbott, 2019; Rice \& Richardson, 2014). However, if maladaptive, it is a primary and secondary contributor to a wide range of psychopathology, physical ailments, and social and occupational strife (Curran \& Hill, 2019; Egan et al., 2011; Fry \& Debats, 2009; Vidic \& Cherup, 2019). It is also linked to numerous physical health ailments through the pathways of stress, such as ulc- 
ers, migraines, chronic fatigue, and hypertension (Molnar et al., 2018).

The purpose of this review is to collate the evidence on the impacts of maladaptive perfectionism and to review the treatment

\subsection{Rationale for Review}

Maladaptive perfectionism can have an insidious and consequential effect on individuals and society; its impacts can be far-reaching and contribute to a wide range of mental and physical health concerns (Egan et al., 2011; Flett et al., 2020; Molnar et al., 2018; Smith et al., 2018). Not only does perfectionism make individuals likely to be more susceptible to physical and mental health disorders, but its perniciousness also results in decreased capacity to cope with hardship and illness (Hewitt \& Flett, 2002). Due to the significant and multi-faceted negative impacts, perfectionism must be targeted for treatment, both as a primary presenting problem and as a related set of symptoms comorbid with other diagnoses (Crosby et al., 2013; Flett et al., 2016; Fry \& Debats, 2009). Increased awareness of perfectionism in the clinical setting and suggestions for evidence-based treatment will provide clinicians the tools needed to assist with this far-reaching and often misunderstood affliction.

\subsection{Purpose of the Study}

The purpose of this review was to explore the existing literature on the effects of maladaptive perfectionism on individuals, synthesize the findings, and provide recommendations for best practices for accompanying treatment. There has been a resurgence since the 1990s in the scientific study of perfectionism, this study highlights a need for continued investigation into this area by clinicians and scholars (Crosby et al., 2013; Hewitt \& Flett, 2002; Smith et al., 2018). A systematic review of the literature was conducted in order to analyze and interpret key themes and discover new meanings. It aimed to provide clarity on the factors aiding the development and maintenance of perfectionism, the detrimental psychological impacts of perfectionism, and the current evidence-based treatments for perfectionism.

\section{Methods}

A systematic review of the literature was chosen for its ability to make new interpretations from existing peer-reviewed research and clarify gaps to promote future. Databases were scanned using a keyword search for studies published between the years 2010-2020. The following databases were searched: PsychArticles, ProQuest, EBSCO Host, Google Scholar, and ProQuest Psychology. Keywords for the search included: perfectionism, maladaptive perfectionism, adaptive perfectionism, locus of control, self-oriented perfectionism, other-oriented perfectionism, socially prescribed perfectionism, cognitive behavioral therapy, self-efficacy, social cognitive theory, and psychodynamic therapy. The majority of studies had sample populations that included adolescents, college students, 
and adults. The Preferred Reporting Items for Systematic Reviews and Meta-Analyses for Protocols Checklist (PRISMA-P) is one of the most commonly used methods of analysis in systematic reviews. PRISMA is widely accepted as a strong tool to ensure methodological quality and was used in this study to determine study inclusion (Gates \& March, 2016; Moher et al., 2009). The articles included in the study were then analyzed thematically in relevance to answering the research questions of the study.

\section{Results}

The search strategy described previously yielded $(\mathrm{n}=118)$ peer-reviewed research papers for inclusion in this review. Rather than describing the individual papers identified in the review a thematic analysis was conducted and is presented below.

The multifaceted interaction between the individual's genetic predispositions and their environment is prevalent across research on perfectionism. It is also supported by Albert Bandura's social cognitive theory (SCT), which is the theoretical framework for this study. Social cognitive theory is an extension of social learning theory (SLT), which explains the imitative power of children's observations of parent behaviors and the negative consequences of insufficient modeling (Stewart \& De George-Walker, 2014; Bandura, 1997; Bandura, 2004). SCT is grounded in the assertion that the individual, behavior, and environment are interconnected and have a reciprocal influence on one another. Human experience encompasses learning and knowledge, which influences behavior, which occurs in specific contexts and environments. Rooted in SLT, SCT is primarily concerned with learning, but also emphasizes self-efficacy and is most commonly used to understand how individuals navigate and experience health related issues (Thojampa \& Sarnkhaowkhom 2019).

Hewitt and Flett's (1991) multi-dimensional theory of perfectionism categorizes perfectionism as having three components that the literature examines individually and as inter-dependent processes: self-oriented, other-oriented, and socially prescribed thinking styles. Self-oriented perfectionism describes the propensity for individuals to set and be driven by self-imposed high standards. Other-oriented perfectionism is the psychological propensity of holding others to unrealistic standards. Socially prescribed perfectionism is the belief that cultural forces and society at large demand standards that cannot be reasonable and consistently met (Hewitt \& Flett, 1991). This review identified that it is generally accepted that this paradigm holds utility for a preponderance of peer-reviewed literature on perfectionism (Walton et al., 2020; Yildiz et al., 2020).

\section{Etiology and Progression}

Research suggests that perfectionism can have a negative effect on all domains of an individual's life and is found amongst all age groups, socio-economic classifications, and ethnicities (Burgess \& Dibartolo, 2016). The rates of perfectionism 
are on the rise; a meta-analysis conducted by Curran and Hill (2019) found perfectionism has increased 32\% in the birth cohort of United States, Canadian, and British students from 1989-2016. There are a range of theories as to how perfectionism develops and is maintained that are informed by research in personality theory, social and cognitive psychology, and epigenetics (Curran \& Hill, 2019; Frost et al., 1990; Hewitt et al., 2018; Smith et al., 2018; Stoeber, 2018). A comprehensive list of themes related to etiology and progression was compiled.

There was a preponderance of literature that focused on the notion that social predicates for the development of perfectionism hinge on high parental expectations. Expectations can manifest in a person's cognitive schema either by way of overt rigid and invalidating behaviors by parents, or imaginal expectations based on a variety of subtle family dynamics and cultural and societal parameters (Curran \& Hill, 2019; Newman et al., 2019; Smith et al., 2018). Associations between parenting style and the development and maintenance of perfectionism were found to be a dominant theme in the literature.

Parenting. Parenting style affects the development and maintenance of perfectionism and is notably compounded when early childhood traits of perfectionistic inclinations are present (Hewitt et al., 2018). The multi-dimensional theory proposes that both intrapersonal and interpersonal factors are present for those experiencing perfectionistic traits. Perfectionism was found to be correlated in multiple studies with parental criticism and high expectations, and the natural consequences of a child perceiving that others expect an unattainable high standard in functioning across a wide range of social, emotional, and academic domains (Stewart \& De George-Walker, 2014; Curran \& Hill, 2019; Frost et al., 1990; Hewitt et al., 2018; Smith et al., 2018; Stoeber, 2018).

Invalidating early childhood experiences can result in insecure attachments, wherein the parent child dyad does not form the bond that encourages an anchor that a child can use to self-explore and grow. This type of attachment in this dyad can contribute to psychological disorders, both independent and associated with maladaptive perfectionism (La Mela et al., 2015). It can decrease perception of self-worth, and therein create feelings of shame, depression, anger, and anxiety (Stewart \& De George-Walker, 2014; Curran \& Hill, 2019; Frost et al., 1990; Hewitt et al., 2018; Smith et al., 2018; Stoeber, 2018). Although the literature is mixed in positing if it is genetic or learned, studies analyzed in this review confirmed the associations between insecure attachment and manifestations of perfectionism.

Curran and Hill (2019) concluded in a longitudinal study that perfectionism appears to be worsening over time. Socially prescribed perfectionism has increased at a rate double of the other domains in the multi-dimensional paradigm. Many theorists suggest that the confluence of rapidly changing social structures including social media's influence on self-oriented and socially oriented perfectionism, is a factor in rising rates of perfectionism as well as the early childhood adoption of perfectionistic behaviors and thinking styles (Burgess \& Di- 
bartolo, 2016; Newman et al., 2019; Sharma, 2016; Smith et al., 2018; Stoeber, 2018; Vidic \& Cherup, 2019).

Cognition. The quantitative studies on the role of cognition in the development of and maintenance of perfectionism were relatively limited and yet cognitive schemas appeared to be part of the historical canon in this area of study and a burgeoning area of interest. It is generally accepted that fixed cognitive states influence perfectionism by way of underlying personality traits and are present in the cognitive schema of those who express the negative impact of perfectionistic rituals and unwanted emotional states (Stewart \& De George-Walker, 2014; Dodd et al., 2019). Self-oriented perfectionism is the propensity for individuals to set and be driven by self-imposed high standards (Molnar et al., 2018). Throughout the literature, it was noted that self-oriented perfectionistic traits are implicated as precursors to the development of a perseverative cognitive style and creates a vulnerability to psychopathology (Burgess \& Dibartolo, 2016; Egan et al., 2014; Molnar et al., 2018; Smith et al., 2018). Also, consistent throughout the literature was the role that social-oriented perfectionism has in the habitual thinking of style of those displaying persistent cognitions of not ever satiating the desire to achieve.

Personality Traits. Early research on perfectionism relied heavily on the discussion of personality traits and temperamental predispositions (Stoeber, 2018). These findings, while not diminishing the role of personality traits, brought a new focus on the differentiation between traits and perfectionistic cognitions. Perfectionistic cognitions are suggested to be a response to daily pressures and their synergistic interaction with a pre-existing trait orientation toward perfectionism (Stoeber, 2018). Tonta and colleagues (2018) suggested that attentional bias for those with maladaptive perfectionism incline individuals toward rapidly focusing on internal and external stimuli. Perfectionists were found to have difficulty removing their focus from the negative perfectionist driven stimuli. The result was that a ruminative style is reinforced.

Numerous other studies concluded that a perfectionistic mindset, or the perfection cognition theory (PCT), influences cognitive thinking styles and biases in perfectionists (Flett et al., 2016; Stoeber, 2018). These factors are manifold and make a person vulnerable to negative self-evaluations and concerns about how they feel others perceive them. In sum, three elements were found to occur across the literature: 1) Perfectionism mediates the speed and occurrence of perseverative cognitions, and this rumination develops into unwanted affective states 2) Perfectionism has a unique form of perseveration where overthinking in regard to day-to-day tasks is the overarching cognitive schema. 3) The overlearned cognitive schema found in perfectionists creates a cognitive memory of past mistakes and marked anxiety about new errors, both perceived and real (Macedo et al. 2017; Stoeber, 2018; Tonta et al., 2018).

\section{Psychological Consequences of Perfectionism}

Perfectionism, as a trait and as a domain-specific construct, is found to be both a 
precursor and a result of a multitude of psychological disorders. The psychological impacts of maladaptive perfectionism have negative and far-reaching impacts on individuals and society (Drieberg et al., 2019; Habke \& Flynn, 2002; Kehayes et al., 2019; Starley, 2019). Not only does perfectionism increase susceptibility to mental illness, but its insidious nature makes mental health diagnoses more challenging to manage when they are persistent and present (Hewitt \& Flett, 2002). A comprehensive list of the effects of maladaptive perfectionism was compiled.

Self-Concept. Research suggests that individuals suffering from perfectionism feel that invalidating parental experiences create a sense that others have a set of high demands and expectation that they are unable to meet (Curran \& Hill, 2019). In an attempt to mediate the negative affective states resulting from this dynamic, children begin to take steps to be perfect and experience feelings of unworthiness and thereby a need to achieve due to an attempt to seek cohesion through feeling competent and worthy (Békés et al., 2015; Hewitt et al., 2017). A child's development of the notion of a fear of failure results in the development of low self-esteem (Segrin et al., 2019). The drive to raise children who are educated to the degree that they can engage in the meritocratic tasks necessary to perform in the modern economy, pressures children to achieve and a deleterious affect ensues for those that are dispositionally vulnerable to the negative impacts of maladaptive perfectionism (Enns et al., 2001).

Aspects of other-oriented and self-oriented perfectionism has been tied to demands from the broader culture (Dodd et al., 2019; Jahng \& Kim, 2019; Lipson et al., 2019; Macedo et al., 2017; Shafran et al., 2016). Hewitt et al. (2017) found that college students with significant levels of other-oriented perfectionism had adversarial interpersonal styles and reported low levels of relationship satisfaction along with the sense of having persistent unfair and punitive interactions with others. High levels of self-oriented perfectionism were indicated in a wide range of psychopathology and have linkages to the similar but nuanced construct of socially prescribed perfectionism.

Socially prescribed perfectionism is the belief that others hold standards for the individual that cannot be reasonable and consistently met and have the net effect of the experience of multiple and consistent perceived events of failure (Curran \& Hill, 2019; Smith et al., 2018). Several studies concluded that this form of perfectionism is especially damaging to self-esteem (Curran \& Hill, 2019; Newman et al., 2019; Sharma, 2016; Smith et al., 2018; Stoeber, 2018; Vidic \& Cherup, 2019). In conjunction with internalized parental expectations of social, academic, and occupational success, studies conclude that other outside social forces such as neo-liberalism and meritocracy exacerbate the social correlates of perfectionism and resulting negative affective, behavioral, and social states (Curran \& Hill, 2019; Norwin, 2018; Smith et al., 2018).

A person's overriding desire to present positively to others is linked to perfectionism (Moate et al., 2019). Socially prescribed perfectionism was found to be 
especially deleterious to one's mental health across multiple disorders (Cowie et al., 2018). Maladaptive perfectionism is correlated in several studies with personality traits of being excessively self-critical and relatedly lacking in self-compassion (Curran \& Hill, 2019; Linnett \& Kibowski, 2020; Moate et al., 2019). This review noted that the traits of being self-critical and not possessing the coping skill of self-compassion were factors across the research in numerous disorders and of particular note in depression and anxiety (Moate et al., 2019; Vanstone \& Hicks, 2019).

A study by Park and Jeong (2015) found that individuals with low self-esteem have significantly higher rates of maladaptive perfectionism than do adaptive perfectionists and non-perfectionists. Another study by Moroz and Dunkley (2015) confirmed the relationship between self-esteem and perfectionism. They went further than previous researchers by concluding that avoidance is not only a by-product of this connection but a coping style that is learned and reinforced by the cognitive style of perfectionists. The resulting coping style has a cascade of negative affective and behavioral states and is implicated in psychopathology and of particular note in depression and suicide (Moroz \& Dunkley, 2015). In addition to psychopathology, interpersonal and occupational conflicts were an area of concern for individuals that have perfectionistic traits and are analogous to low self-esteem.

Depression. Research has demonstrated a causative link between depression and perfectionism (Asseraf \& Vaillancourt, 2015; Besser et al., 2020). Factors generally accepted in the literature included social, cognitive, behavioral, personality traits, and environmental influences (Park \& Jeong, 2015). Although maladaptive perfectionism is trans-diagnostic, its presence has been demonstrated to predict baseline depressive traits and similar thoughts of hopelessness (Glover et al., 2007). As discussed, the multi-dimensional framework, and in particular socially prescribed perfectionism, are indicated in both the development and maintenance of depressive symptoms in perfectionists (Moroz \& Dunkley, 2015; Malinowski et al., 2017; Smith et al., 2018).

A study by Asseraf and Vaillancourt (2015) found that middle school students with high scores in socially-prescribed perfectionism had rates of depression that exceeded those with a predominantly elevated score on the self-oriented scale. Similarly, Limburg et al. (2017) found that having perfectionistic strivings was not a predictor for depression; however, having perfectionistic concerns (as in socially prescribed perfectionism) did correlate to a range of psychopathology and, in particular, depression. These results were found in numerous other studies, and while using different terminology, the overwhelming conclusions were that fears of performing to real or imagined standards which results in shame, low-self-esteem, and diminished ability to cope with negative emotions and ultimately making one vulnerable to depression (Cooks \& Ciesla, 2019; Curran \& Hill, 2019; Macedo et al., 2017; Malinowski et al., 2017). Additionally, the circular nature of the insidious effect of a socially-prescribed perfectionism ruminative style, 
independent of self-esteem, reduce the coping mechanism of self-compassion. Self-compassion was determined to be protective of depression and a lack thereof present in perfectionists (Ferrari et al., 2018; Macedo et al., 2017; Mehr \& Adams, 2016).

Suicide. Some researchers in the field of suicidality agree that suicide is a multi-factorial process, and it generally understood that negative cognitions are a precursor to suicide (Kiamnesh et al., 2015). What is less understood in the interplay between perfectionism and suicidal ideation (Shahnaz et al., 2018; Smith et al., 2018). An initial study by O'Connor (2007) has been instrumental for subsequent research. The findings did not make the overt claim that perfectionism was linked to suicide, but the study did conclude that self-critical evaluations are strongly associated with suicidality in adults. O'Connor's (2007) conception of what is meant by self-critical evaluations aligns with the elements of the multi-dimensional theory. Muyan and Chang (2015) drew a more definite finding by asserting that depression and suicide are well established in the literature. Therefore, perfectionism can reasonably be a factor in the complex machinations of suicidal cognitions and behaviors. They concluded that university students who presented with both perfectionism and suicidal ideation had two salient dimensions in common: 1) reports of a history of parental criticism and 2) doubts about actions (Muyan \& Chang, 2015). Several studies noted a range of dimensions associated with low self-esteem, self-criticism, and suicidal ideation (Campos et al., 2018).

Although much of the literature reviewed and analyzed used adolescents as the primary participants, the perfectionist/suicide link was also found to be a factor in adult men. Kiamnesh et al. (2015) did an extensive analysis of men who died by suicide aged 22 to 58 who had no previous history of mental illness or suicidal history. They concluded that three themes emerged as common factors: a cracking façade, a total loss of coping ability, and a total escape. These dimensions and their accompanying definitions were aligned with the other researchers stating that esteem is a recursive variable in the suicide/perfectionism linkage. Since individuals with maladaptive perfectionistic traits can be overly concerned with putting on a competent façade, other more overt signs of mental illness typically associated with precursors of suicide are not visible. The lack of visible signs consistent with suicidal risk put perfectionists at higher risk for suicide (Kiamnesh et al., 2015). This association was validated it was noted that all variables associated with perfectionistic cognitions (with the exception of other-oriented perfectionism) are associated with a higher risk for suicide (Shahnaz et al., 2018, Stoeber, 2018).

What was ascertained through a review of the literature was the preponderance of evidence suggesting both the trans-diagnostic nature of perfectionism and the sense of hopelessness that persons can experience. Moreover, the lowering of self-esteem and shame appeared to be so profound that the consequence of dying by suicide was a likely outcome, wherein the desire to keep the illusion 
of being perfect amplifies the danger of perfectionism as this means that overt signs are often not apparent. Finally, there was one additional area regarding the theme of self-esteem and perfectionism. The role that early perfectionism can arise in the formation of a subsequent diagnosis of personality disorders. Recent interest has piqued in this regard, and several studies have concluded that maladaptive perfectionism is a pervasive factor thought not emphasized in routine assessment (Bigaier, 2019; Dimaggio et al., 2015; Limburg et al., 2017). In particular, concern about one's actions and worry about mistakes are indicated and predictive for a wide range of clinically significant personality disorders (Dimaggio et al., 2015). Notably, other-oriented perfectionism had an especially strong relationship with disorders involving grandiosity, as did narcissism, a disorder whereby a person seeks to sustain a self-presentation of perfection. While attempting to maintain one's imagined façade of perfection for what is deemed needed for others' approval, the competing force of low self-esteem fluctuates from being both a covert and overt cognitive schema (Casale et al., 2016).

Anxiety. The literature suggests that perfectionism and anxiety are linked, and anxiety sensitivity is a predictor for pathology both as a primary and secondary diagnosis (Burgess \& Dibartolo, 2016; Diaz, 2018; Damian et al., 2017; Frost \& DiBartolo, 2002; Mohammadian et al., 2018; Smith et al., 2018). Perfectionism can be responsible for fast and negatively charged cognitions, which manifests as rumination, and rumination in turn becomes perseveration. The dynamic nature of these cognitive and behavioral forces can then transform into clinically diagnostic syndromes such as obsessive-compulsive disorder (OCD), panic disorder, and specific phobia (Békés et al., 2015; Dodd et al., 2019).

The overdeveloped ruminative pattern imprints a cognitive memory of past failures and anxiety about the notion that new errors will be made (Mohammadian et al., 2018). Another study by Akram et al. (2015) bolstered the causal relationship between these variables and additionally found that insomnia and depression were often co-morbid in the anxiety, rumination, and perfectionism triad. Additionally, and consistent with themes found throughout this project, the authors noted the marked anxiety in those individuals with the highest scores in socially-prescribed perfectionism (Akram et al., 2015).

Erozkan (2016) identified six key areas that are complicit in a positive association with anxiety and perfectionism: doubts about actions, high personal standards, high parental expectations, and a tendency to perceive one's parents as being critical. Damian et al. (2017) also found that individuals who displayed perfectionist strivings coupled with marked perfectionistic concerns were a risk factor for maladaptive ruminative thinking and inherently present in those who display perfectionistic personality traits.

Burgess \& Dibartolo (2016), in their in-depth study, found that the anxietyperfectionism link is mediated and moderated via three domains: stress/emotion regulation, social factors, and cognition. They concluded that high standards for 
self-evaluation may increase subjective and objective stress, isolation, and inability to cope (Burgess \& Dibartolo, 2016).

Eating Disorders. In addition to the anxiety-related diagnosis that is analogous to the finding of the studies reviewed, another serious set of cognitions and behaviors was found in this review-disordered eating. While there is broad agreement that an eating disorder-perfectionism link exists, the quantitative analysis demonstrating a causal relationship is relatively limited in the literature (Kehayes et al., 2019; La Mela et al., 2015; Stoeber \& Yang, 2015; Wade et al., 2016). Despite the limits in the research, numerous studies have concluded by way of qualitative analysis that eating disordered thinking styles share traits and have associations with those showing high levels of maladaptive perfectionism (Barnes \& Caltabiano, 2017; Kehayes et al., 2019; La Mela et al., 2015; Wade et al., 2016). Similar to the finding discussed previously in regard to anxiety, La Mela et al. (2015) found that individuals with high parental concerns and perceived criticism were more likely to exhibit eating disordered behaviors.

Stoeber and Yang (2015) examined the relationship between what they termed physical appearance perfectionism, which is a perseverative cognitive focus on one's appearance that impairs other life domains. Their findings demonstrated a similar but nuanced relationship between perfectionism and eating-disordered thinking (Stoeber \& Yang, 2015). Barnes and Caltabiano (2017) examined a syndrome called orthorexia, which is cognitive focus on eating what is perceived to be healthy foods. The conclusions of their study show that self-oriented perfectionism can be correlated with orthorexia, and therefore, suggest that perfectionism, in general, is a risk factor for the development and maintenance of eating disorders (Stoeber \& Yang, 2015). Santos et al. (2018) advanced the linkages of the multi-dimensional paradigm of perfectionism and highlighted that the family environment was a significant factor in both perfectionism and eating disorders. Drieberg et al. (2019) emphasized the trans-diagnostic nature of depression and anxiety and perfectionism and posits that eating disorders can have both a direct and indirect relationship to perfectionism due to the bi-directionality of perfectionism and related disorders (Drieberg et al., 2019). Therefore, this information suggests a synergistic process of personality traits alongside the environment that links self-oriented perfectionism and its pernicious ruminative cognitive style.

\section{Available Treatments}

In general, the research demonstrated that the targeted intervention of perfectionism as an isolated issue is a burgeoning area, and the number of available treatments is limited. The predominant intervention that has undergone research and implementation in the clinical setting is cognitive-behavioral therapy (CBT) and numerous recent studies have emerged examining CBT in individual, group, and internet-based formats (Egan \& Hine, 2008; Egan et al., 2011; Shafran et al., 2017). Perfectionism was commonly discussed in the psychological litera- 
ture as a personality trait, and therefore, there is preliminary evidence, albeit limited in scope, that longer-term psychodynamic approaches may be efficacious. More recent interest in mindfulness-based approaches is emergent, as is some limited support for various self-help interventions (Linnett \& Kibowski, 2020; Mehr \& Adams, 2016; Ong et al., 2019).

Evidence-Based Treatment. The deleterious effects of perfectionism were trans-diagnostic, and the treatments to date have been limited. Dibartolo et al. (2002) conducted the first study as to whether cognitive behavioral therapy was effective in treating perfectionism. The participants in the study had all scored high on the concern over mistakes domain per the Multi-Dimensional Model and accompanying Frost Multi-Dimensional Perfectionism Scale (FMDPS). By using the cognitive model as a theoretical lens, the authors found that by using the technique of cognitive restructuring, participants were able to lessen negative cognitions and resultant unwanted emotions in perfectionists. In another study influenced by Dibartolo et al. (2002), Shahar (2006) utilized the Multi-dimensional Perfectionism Scale (MPS) to select participants with scores in the clinically significant range along with several validated scales for depression. By using a cognitive intervention to restructure thoughts related to personal standards, it was demonstrated that post-treatment outcomes had improved scores and results.

Glover et al. (2007) designed and tested a 10-week CBT manualized protocol. The treatment encompassed four specific CBT treatment components which others would go on to emulate and adapt in varied manifestations: 1) a personalized formulation in terms of clinical perfectionism; 2) broadening the patient's scheme for self-evaluation; 3) using behavioral experiments to test competing hypotheses; and 4) using cognitive behavioral methods to address personal standards, self-criticism and cognitive biases that maintain clinical perfectionism (Glover et al., 2007).

Although not a randomized controlled trial, participants had clinically significant improvement, and a three-month follow-up also indicated a lasting positive effect of the CBT intervention. This study extended the previous work by Shahar (2006) by suggesting not only the efficacy but the possibility of longstanding effects of a CBT protocol for perfectionism.

Several authors used the initial finding of CBT's effectiveness for perfectionism as means to develop and test similar protocols in self-help iterations and web-based applications that utilize variations with and without adjunctive coaching by therapists (Kothari et al., 2019; Rozental et al., 2017; Valentine et al., 2018; Zetterberg et al., 2019). Across the range of web-based and self-help CBT protocols for perfectionism, the literature indicates a positive effect but without any noted advantage from one design to another (including those that utilize in-person adjunctive coaching). The most recent findings by Kothari et al. (2019) report that internet-based cognitive behavioral therapy reduces underlying processes related to perfectionism, such as self-esteem and self-compassion. This finding points toward the possibility that a change in one variable along the 
trans-diagnostic continuum can lead to change, amongst other related symptoms.

Emerging Treatments. The research revealed that cognitive-behavioral therapy was the most prominent and widely researched method for treating perfectionism. However, there was also an inchoate drive toward utilizing mindfulness-based approaches to treat perfectionism directly and to alleviate the concomitant symptoms across the trans-diagnostic spectrum. Wimberley et al. (2016) conducted the first study, which suggested that mindfulness may treat perfectionism when paired with existing mindfulness-based approaches for depression and anxiety. James and Rimes (2017) found an increase in self-compassion through a mindfulness-based cognitive therapy group and that daily perfectionistic cognitions were reduced, suggesting that mindfulness could be a potentially efficacious treatment for perfectionism. The research also revealed that mindfulness lessens the cognitive and emotional load present for those who feel a perfectionistic self-presentation is necessary to maintain for others (Flett et al., 2020).

Ong et al (2019) concluded that participants in a mindfulness-based group protocol experienced better improvements in perfectionism and related disorders, such as depression and anxiety than the waitlist control group. Research also supported that in addition to improvements in perfectionism domains, the mindfulness interventions directly target the perfectionistic cognitions of individuals suffering from the ill effects of this disorder (Ong et al., 2019; Vidic \& Cherup, 2019).

Literature regarding treatment for perfectionism was not wide-ranging, and areas of treatment that have been tested and do exist in the literature were dominated by CBT. The literature posits that a psychodynamic approach is a potentially viable intervention to treat perfectionism (Hewitt et al., 2017; Rice \& Preusser, 2002). The notion being that a focus on the resolution of relational issues that are inherent in maladaptive perfectionism may have a beneficial effect on other disorders, such as depression and anxiety.

In the first of its kind study, Hewitt et al. (2015) demonstrated that modest reductions in perfectionism scales were observed. Their findings, while provisional and limited in size and scope, may lead to future research in this area of study. Additionally, Hewitt et al. (2015) found that the relational nature of psychodynamic therapy and its focus on what is deemed in the analytic tradition as therapy interfering behaviors was another benefit of this approach. A psychodynamic therapeutic orientation is one that encourages a strong alliance between therapist and client and therefore increases treatment compliance and persistence. The unique focus on the therapeutic alliance was found to be of import as perfectionistic behaviors can interfere with treatment planning and implementation (Hewitt et al., 2017).

\section{Summary}

Perfectionism can be a risk factor associated with a myriad of adverse health ef- 
fects associated with it; unfortunately, this trait is likely increasing in society and can affect different age groups, ethnicities, and socio-economic classes.

The deleterious effects of maladaptive perfectionism are multitudinous and associated with decreased psychological and physical health (Molnar et al., 2018). Significant psychological effects negatively impacting multiple domains of an individual's life and results in an increase of persons presenting for psychological treatment. Understanding factors driving the increase is important. Some notable factors include: the interwoven set of genetics, trends in personality traits, increased standards for academic achievement, globalization and comparison, cultural diversity and exposure to different communities and individuals, work related demands resulting in increased burnout, and shifting social norms and means of connecting with others (Curran \& Hill, 2019; Damian et al., 2017; Sedera \& Lokuge, 2020; Smith et al., 2018).

The purpose of this study was to investigate the psychological and physical impacts of maladaptive perfectionism on individuals, and best practices for accompanying treatment. Because perfectionism has significant negative psychological consequences impacting individuals and their families, assessing and targeting its symptoms could benefit the individual and society. Increased awareness of perfectionism in the clinical setting and continued focus on developing and employing evidence-based treatment will provide clinicians the tools needed to assist with this far-reaching and often little-known affliction. This systematic review of the literature focused on synthesizing the literature to better understand development, impact, and treatment for the trait.

\section{Findings}

This review confirmed that maladaptive perfectionism is associated with a myriad of negative psychological consequences. While the research toward finding effective treatments that uniquely target perfectionism is in the initial stages, there is evidence that cognitive-behavioral therapy (CBT) is an effective form of treatment and that several other inchoate therapies hold promise (Shafran et al., 2016). In addition to CBT, there was preliminary evidence, albeit limited, that longer-term psychodynamic approaches may yield efficacious results for perfectionism (Hewitt et al., 2017). Mindfulness-based approaches likewise were an emergent treatment currently being studied, as are burgeoning self-help interventions that combine $\mathrm{CBT}$ and mindfulness-based interventions (Linnett \& Kibowski, 2020; Mehr \& Adams, 2016; Ong et al., 2019).

According to the trait theory of personality, perfectionism is both a result of and the cause of a multitude of psychological disorders (Drieberg et al., 2019). More research is needed on the often-veiled cluster of symptoms associated with perfectionism in order to add more information to the growing, albeit limited, data on this topic. Additionally, it is not clear if and how clinicians screen for perfectionism in their initial intake session and if this results in misguided treatment planning. Although there has been an increase in the research related 
to perfectionism, not enough is being done to assess and target the devastating impacts of this often-veiled malady (Smith et al., 2017). The scientific study of perfectionism is not new, more can and needs to be done to support clinicians in identification and treatment and to combat the adverse and manifold effects of this personality trait.

\section{Implications for Professional Practice}

The incidence of perfectionism continues to increase, and therefore, the negative consequences are compounded and felt throughout society (Curran \& Hill, 2019). Although there has been an increase in the research related to perfectionism, not enough is being done to assess and target the devastating impacts of this often-veiled malady (Smith et al., 2018). By increasing awareness of the complicated nature of perfectionism in clinicians, educators, and parents, it is more possible to intervene early and prevent the suffering that would otherwise go unabated.

Although interventions are available, the relative lack of specificity in practical clinical settings is an area for improvement. Focusing on the utility of accurately assessing perfectionism and its related symptomology, researchers can prioritize the need to develop assessment measures and guidance. Understanding the underlying processes of perfectionism could have benefits to clinicians and clients alike. This study elucidates several areas that may be worthwhile to consider regarding intervention, treatment, and assessment. Understanding the predicates and influences of perfectionism does not equal effective treatment. It is known that other-oriented, self-oriented, and socially-oriented perfectionistic traits have their subtle effects; however, targeted treatments need to be developed beyond treating perfectionism as a symptom related to associated disorders like depression and anxiety and eating disorders, and when appropriate, perfectionism needs to be understood as having the power to be the primary disorder driving symptoms of other disorders. Although this study highlights the finding that perfectionism is a trans-diagnostic process, not treating perfectionism as a primary diagnosis may result in inadequate treatment compliance (Hewitt et al., 2017).

Early and specific interventions also appear to hold the key to effectively stemming the pernicious nature of maladaptive perfectionism. Schools could develop and implement early evidence-based practices from the existing cognitive-behavioral and mindfulness-based practices in order to build the coping strategies and self-compassion necessary to deal with the stealthy nature of the initial stages of perfectionism. Programmatic efforts in higher education would also prove beneficial. The pressures faced by college students are unique and act to dynamically affect those who have perfectionistic traits and catalyze a new range of symptoms for those whose perfectionism is newly set into motion by these demands.

As has been discussed extensively in this study, early childhood invalidation 
and related asynchrony is a foundational element in the epigenetic nature of perfectionism (Stoeber \& Yang, 2015). Increasing parental awareness of the deleterious and long-term consequences of perfectionism can also be vitally important. Relatedly, the development of evidence-based programs to assist with interventions for parents who, unwittingly or not, create an environment rife with unattainable expectations may be pivotal in preventing maladaptive perfectionism. Parental intervention programs may also play a key role in assisting in reinforcing the positive skills that a child learns from therapeutic perfectionism interventions.

\section{Recommendations for Research}

This systematic review highlighted the importance of several future directions for researching the psychological impact of perfectionism and accompanying treatment. A common theme in the literature was the necessity for more research in all facets of perfectionism and mental health. Additional awareness of and assessment measures for perfectionism in clinical settings was underexplored. Socially prescribed perfectionism is a particularly new and therefore understudied area. The confluence of social and economic pressures, which are heightened by social media and increased parental demands, is an area that could benefit from focused research on the moderating and mediating variables, which in turn can inform new and improved forms of treatment.

Future research could focus on the assessment of perfectionism in clinical settings and how this transitions to treatment planning and intervention. By not directly addressing the perfectionistic nature of the behavioral and cognitive complaints, it is likely that other treatment goals will be, in turn, compromised (Hewitt et al., 2018). Therefore, early assessment in the initial stages of treatment conceptualization will yield valuable information to help determine when perfectionism is most effectively prioritized. In addition to greater treatment compliance, overcoming the resistance to consistent engagement in the therapeutic process due to confounding elements of perfectionism may offer a more significant effect and more durable symptom reduction.

Further investigation into the role that a steady increase in social demands and familial pressures play in maladaptive perfectionism may inform and benefit all areas of intervention along with primary and tertiary prevention. As this study elucidates, in addition to perfectionism's association with a wide range of psychological disorders, its relative increase over time necessitates continued focus and attention. This study revealed the importance of directly integrating programmatic efforts based on the best scientific information available and encouraging therapeutic and educational settings to treat perfectionism not merely as a symptom of other disorders, but when valid, the primary reason behind other related cognitive and behavioral concerns.

Another broad area highlighted by this study's literature review is the lack of substantive information on what cultural aspects are essential for assessing and 
treating perfectionism. Given that culturally competent therapy is foundational for psycho-social assessments, increased awareness about how cultural aspects of the individual and family impacts perfectionism and its outward manifestation is warranted. The research would benefit from studies comparing a range of cultural and socio-economic variables in order to better determine the individual factors influencing perfectionism from a cultural and economic lens. Additionally, as most research to date focuses on participants from countries where individualism in prized, comparing and contrasting any variance from individualistic versus collectivistic cultures is warranted.

Although cognitive-behavioral therapy (CBT) has been shown to be useful for perfectionism, continued studies with randomized controls and large sample sizes will benefit researchers by providing increased information about the efficacy and what elements of the therapy are most beneficial. Relatedly, while several scales are available for assessing perfectionism, the continued research toward finding reliable, valid, and brief scales, could be a benefit for clinicians to screen for perfectionism and therefore help to focus treatment accordingly.

\section{Conclusion}

This systematic review of literature collected, analyzed, and synthesized peer-reviewed literature on the negative psychological impacts of perfectionism and accompanying treatments. The study endeavored to understand the particularities underlying the development and maintenance of maladaptive perfectionism and sought to understand what evidence-based practices are available for the treatment of this far-reaching emotional and behavioral affliction. The review identified the following gaps: clinical screening of perfectionism and accompanying treatment planning, differentiating perfectionism as a standalone presenting problem or a specifier to comorbid conditions, and efficacy of treatment approaches specific to perfectionism as the primary presenting problem.

Perfectionism is a complex personality trait and increases risk for susceptibility to multiple physical and mental health effects; the increase of its presence in society underscores the complex and inter-related nature of genetic predisposition, human nature and behavior, and the environment. Although perfectionism has been discussed in the literature going back to the historical canon of psychology, only recently has there been a relative resurgence of interest in this topic. Similarly, the research related to effective treatments and assessment in the clinical setting is inchoate. In focusing on the negative psychological consequences coupled with ongoing research, numerous avenues are open to researchers and mental health clinicians for the continued formation of new and innovative prevention and intervention measures for this complex issue.

\section{Conflicts of Interest}

The authors declare no conflicts of interest regarding the publication of this paper. 


\section{References}

Akay, S., \& Bratton, S. (2017). The Effects of Adlerian Play Therapy on Maladaptive Perfectionism and Anxiety in Children: A Single Case Design. International Journal of Play Therapy, 26, 96-110. https://doi.org/10.1037/pla0000043

Akram, U., Ellis, J. G., \& Barclay, N. L. (2015). Anxiety Mediates the Relationship between Perfectionism and Insomnia Symptoms: A Longitudinal Study. PLoS ONE, 10, e0138865. https://doi.org/10.1371/journal.pone.0138865

Asseraf, M., \& Vaillancourt, T. (2015). Longitudinal Links between Perfectionism and Depression in Children. Journal of Abnormal Child Psychology, 43, 895-908. https://doi.org/10.1007/s10802-014-9947-9

Bandura, A. (1997). Self-Efficacy: The Exercise of Control. New York: Henry Holt \& Co.

Bandura, A. (2004). Health Promotion by Social Cognitive Means. Health Education and Behavior, 31, 143-164. https://doi.org/10.1177/1090198104263660

Barnes, M. A., \& Caltabiano, M. L. (2017). The Interrelationship between Orthorexia Nervosa, Perfectionism, Body Image and Attachment Style. Eating and Weight Disorders, 22, 177-184. https://doi.org/10.1007/s40519-016-0280-x

Békés, V., Dunkley, D. M., Taylor, G., Zuroff, D. C. et al. (2015). Chronic Stress and Attenuated Improvement in Depression over 1 Year: The Moderating Role of Perfectionism. Behavior Therapy, 46, 478-492. https://doi.org/10.1016/j.beth.2015.02.003

Besser, A., Flett, G. L., Sherry, S. B., \& Hewitt, P. L. (2020). Are Perfectionist Thoughts an Antecedent or a Consequence of Depressive Symptoms? A Cross-Lagged Analysis of Perfectionism Cognitions Inventory. Journal of Psychoeducational Assessment, 38, 99-111. https://doi.org/10.1177/0734282919877764

Bigaier, S. (2019). Acceptance and Commitment Therapy for the Treatment of Perfectionism. Dissertation Abstracts International: Section A. Humanities and Social Sciences.

Burgess, A. M., \& Dibartolo, P. M. (2016). Anxiety and Perfectionism: Relationships, Mechanisms, and Conditions. In F. M. Sirois, \& D. S. Molnar (Eds.), Perfectionism, Health, and Well-Being (pp. 177-203). Cham: Springer.

https://doi.org/10.1007/978-3-319-18582-8_8

Campos, R. C., Holden, R. R., Baleizao, C., Cacador, B., \& Fragata, A. S. (2018). Self-Criticism, Neediness, and Distress in the Prediction of Suicide Ideation: Results from Cross-Sectional and Longitudinal Studies. The Journal of Psychology, 152, 237-255. https://doi.org/10.1080/00223980.2018.1446895

Casale, S., Rugai, L., Flett, G. L., \& Hewitt, P. L. (2016). The Interpersonal Expression of Perfectionism among Grandiose and Vulnerable Narcissists: Perfectionistic Self-Presentation, Effortless Perfection, and the Ability to Seem Perfect. Personality and Individual Differences, 99, 320-324. https://doi.org/10.1016/j.paid.2016.05.026

Cooks, J. A., \& Ciesla, J. A. (2019). The Impact of Perfectionism, Performance Feedback, and Stress on Affect and Depressive Symptoms. Personality and Individual Differences, 146, 62-67. https://doi.org/10.1016/j.paid.2019.03.048

Cowie, M. E., Sherry, S. B., Hewitt, P. L., \& Flett, G. L. (2018). Perfectionism and Academic Difficulties in Graduate Students: Testing Incremental Prediction and Gender Modification. Personality and Individual Differences, 123, 223-228.

https://doi.org/10.1016/j.paid.2017.11.027

Crosby, J., Nafziger, M., \& Twohig, M. (2013). Using Acceptance and Commitment Therapy (ACT) to Treat Perfectionism in College Students. In J. Pistorello (Ed.), Mindfulness and Acceptance for Counseling College Students (pp. 140-158). Oakland, CA: New Harbinger Inc. 
Curran, T., \& Hill, A. P. (2019). Perfectionism Is Increasing over Time: A Meta-Analysis of Birth Cohort Differences from 1989 to 2016. Psychological Bulletin, 145, 410-429. https://doi.org/10.1037/bul0000138

Damian, L. E., Negru-Subtirica, J., Stoeber, J. \& Băban, A. (2017). Perfectionistic Concerns Predict Increases in Adolescents' Anxiety Symptoms: A Three-Wave Longitudinal Study. Anxiety, Stress, and Coping, 30, 551-561. https://doi.org/10.1080/10615806.2016.1271877

De Cuyper, K., Claes, L., Hermans, D., Pieters, G., \& Smits, D., (2015) Psychometric Properties of the Multidimensional Perfectionism Scale of Hewitt in a Dutch-Speaking Sample: Associations with the Big Five Personality Traits. Journal of Personality Assessment, 97, 182-190. https://doi.org/10.1080/00223891.2014.963591

Diaz, F. M. (2018). Relationships among Meditation, Perfectionism, Mindfulness, and Performance Anxiety among Collegiate Music Students. Journal of Research in Music Education, 66, 150-167. https://doi.org/10.1177/0022429418765447

Dibartolo, P. M., Frost, R. O., Dixon, A., \& Almodovar, S. (2002). Can Cognitive Restructuring Reduce the Disruption Associated with Perfectionistic Concerns? Behavior Therapy, 32, 167-184. https://doi.org/10.1016/S0005-7894(01)80051-4

Dimaggio, G., Lysaker, P. H., Calarco, T., Pedone, R., Marsigli, N., Riccardi, I., Sabatelli, B., Carcione, A., \& Paviglianiti, A. (2015). Perfectionism and Personality Disorders as Predictors of Symptoms and Interpersonal Problems. American Journal of Psychotherapy, 69, 317-330. https://doi.org/10.1176/appi.psychotherapy.2015.69.3.317

Dodd, D. R., Parsons, E. M., Clerkin, E. M., Forest, L. N., Velkoff, E. A., Kuntsman, J. W., \& Smith, A. R. (2019). The Use of Cognitive Bias Modification to Reduce Perfectionism. Journal of Behavior Therapy and Experimental Psychiatry, 64, 167-174. https://doi.org/10.1016/j.jbtep.2019.04.002

Drieberg, H., McEvoy, P. M., Hoiles, K. J., Shu, C. Y., \& Egan, S. J. (2019). An Examination of Direct, Indirect and Reciprocal Relationships between Perfectionism, Eating Disorder Symptoms, Anxiety, and Depression in Children and Adolescents with Eating Disorders. Eating Behaviors, 32, 55-59. https://doi.org/10.1016/j.eatbeh.2018.12.002

Egan, S. J., \& Hine, P. (2008). Cognitive Behavioral Treatment of Perfectionism: A Single Case Experimental Design Series. Behaviour Change, 25, 245-258. https://doi.org/10.1375/bech.25.4.245

Egan, S. J., van Noort, E., Chee, A., Kane, A., Hoiles, K. J., Shafran, R., \& Wade, T. D. (2014). A Randomized Controlled Trial of Face to Face Pure Online Self-Help Cognitive Behavioural Treatment for Perfectionism. Behaviour Research and Therapy, 63, 107-113. https://doi.org/10.1016/j.brat.2014.09.009

Egan, S. J., Wade, T. D., \& Shafran, R. (2011). Perfectionism as a Trans-Diagnostic Process: A Clinical Review. Clinical Psychology Review, 31, 203-212. https://doi.org/10.1016/j.cpr.2010.04.009

Enns, M., Sareen, J., \& Freeman, P. (2001). Adaptive and Maladaptive Perfectionism in Medical Students: A Longitudinal Investigation. Medical Education, 35, 1034-1042.

Erozkan, A. (2016). Understanding the Role of Dimensions of Perfectionism on Anxiety Sensitivity. Universal Journal of Educational Research, 4, 1652-1659. https://doi.org/10.13189/ujer.2016.040717

Ferrari, M., Yap, K., Scott, N., Einstein, D. A., \& Ciarrochi, J. (2018). Self-Compassion Moderates the Perfectionism and Depression Link in Both Adolescence and Adulthood. PLOS ONE, 13, e0192022. https://doi.org/10.1371/journal.pone.0192022

Flett, G. L., Nepon, T., \& Hewitt, P. L. (2016). Perfectionism, Worry, and Rumination in Health and Mental Health: A Review and a Conceptual Framework for a Cognitive 
Theory of Perfectionism. In F. M. Sirois, \& D. S. Molnar (Eds.), Perfectionism, Health, and Wellbeing (pp. 121-156). Cham: Springer. https://doi.org/10.1007/978-3-319-18582-8_6

Flett, G. L., \& Hewitt, P. L. (2019). Reflections on Three Decades of Research on Multidimensional Perfectionism: An Introduction to the Special Issue on Further Advances in the Assessment of Perfectionism. Journal of Psychoeducational Assessment, 38, 3-14. https://doi.org/10.1177/0734282919881928

Flett, G. L., Blankstein, K. R., Hewitt, P. L., \& Koledin, S. (1992). Components of Perfectionism and Procrastination in College Students. Social Behavior and Personality, 20, 85-94. https://doi.org/10.2224/sbp.1992.20.2.85

Flett, G. L., Hewitt, P. L., Nepon, T., \& Besser, A. (2017). Perfectionism Cognition Theory: The Cognitive Side of Perfectionism. In J. Stoeber (Ed.), The Psychology of Perfectionism: Theory, Research, Applications (pp. 89-110). London: Routledge. https://doi.org/10.4324/9781315536255-7

Flett, G., Nepon, T., Hewitt, P. L., \& Rose, A. (2020). Why Perfectionism Is Antithetical to Mindfulness: A Conceptual and Empirical Analysis and Consideration of Treatment Implications. International Journal of Mental Health and Addiction, 1-21. https://doi.org/10.1007/s11469-020-00252-w

Frost, R. O., \& DiBartolo, P. M. (2002). Perfectionism, Anxiety, and Obsessive-Compulsive Disorder. In G. L. Fleet, \& P. L. Hewitt (Eds.), Perfectionism: Theory, Research, and Treatment (pp. 341-371). Washington DC: American Psychological Association. https://doi.org/10.1037/10458-014

Frost, R. O., Marten, P., Lahart, C., \& Rosenblate, R. (1990). The Dimensions of Perfectionism. Cognitive Therapy and Research, 14, 449-468. https://doi.org/10.1007/BF01172967

Fry, P., \& Debats, D. L. (2009). Perfectionism and the Five Factor Personality Traits as Predictors of Mortality in Older Adults. Journal of Health Psychology, 14, 513-524. https://doi.org/10.1177/1359105309103571

Gates, N. J., \& March, E. G. (2016). A Neruopsychologist's Guide to Undertaking a Systematic Review for Publication: Making the Most of PRISMA Guidelines. Neuropsychology Review, 26, 109-120. https://doi.org/10.1007/s11065-016-9318-0

Glover, D. S., Brown, G. P., Fairburn, C. G., \& Shafran, R. (2007). A Preliminary Evaluation of Cognitive-Behaviour Therapy for Clinical Perfectionism: A Case Series. British Journal of Clinical Psychology, 46, 85-94.

Habke, A. M., \& Flynn, C. A. (2002). Interpersonal Aspects of Trait Perfectionism. In G. L. Flett, \& P. L. Hewitt (Eds.), Perfectionism: Theory, Research, and Treatment (pp. 151-180). Washington DC: American Psychological Association. https://psycnet.apa.org/doi/10.1037/10458-006 https://doi.org/10.1037/10458-006

Hewitt, P. L., \& Flett, G. L. (1991). Perfectionism in the Self and Social Contexts: Conceptualization, Assessment, and Association with Psychopathology. Journal of Personality and Social Psychology, 60, 456-470. https://doi.org/10.1037/0022-3514.60.3.456

Hewitt, P. L., \& Flett, G. L. (2002). Perfectionism and Stress Processes in Psychopathology. In G. L. Flett, \& P. L. Hewitt (Eds.), Perfectionism: Theory, Research, and Treatment (pp. 255-284). Washington DC: American Psychological Association. https://doi.org/10.1037/10458-011

Hewitt, P. L., Flett, G. L., \& Mikail, S. F. (2017). Perfectionism: A Relational Approach to Conceptualization, Assessment, and Treatment. New York: Guilford.

Hewitt, P. L., Flett, G. L., Mikail, S. F., Kealy, D., \& Zhang, L. C. (2018). Perfectionism in 
the Therapeutic Context: The Perfectionism Social Disconnection Model. In J. Stoeber (Ed.), The Psychology of Perfectionism. London: Routledge.

https://doi.org/10.4324/9781315536255-20

Hewitt, P. L., Mikail, S. F., Flett, G. L., Tasca, G. A., Flynn, C. A., Deng, X., Kaldas, J., \& Chen, C. (2015). Psychodynamic/Interpersonal Group Psychotherapy for Perfectionism: Evaluating the Effectiveness of a Short-Term Treatment. Psychotherapy, 52, 205-217. https://doi.org/10.1037/pst0000016

Jahng, K. E., \& Kim, D. (2019). College Students' Adult Attachment and Career Adaptability: Mediation by Maladaptive Perfectionism and Moderation by Gender. Journal of Career Development, 1-14.

James, K., \& Rimes, K. A. (2017). Mindfulness-Based Cognitive Therapy versus Pure Cognitive Behavioural Self-Help for Perfectionism: A Pilot Randomized Study. Mindfulness, 9, 801-814. https://doi.org/10.1007/s12671-017-0817-8

Kehayes, I. L., Smith, M. M., Sherry, S. B., Vidovic, V., \& Saklofske, D. H. (2019). Are Perfectionism Dimensions Risk Factors for Bulimic Symptoms? A Meta-Analysis of Longitudinal Studies. Personality and Individual Differences, 138, 117-125. https://doi.org/10.1016/j.paid.2018.09.022

Kiamnesh, P., Dieserud, G., \& Haavind, H. (2015). From a Cracking Facade to a Total Escape: Maladaptive Perfectionism and Suicide. Death Studies, 39, 316-322. https://doi.org/10.1080/07481187.2014.946625

Kothari, R., Barker, C., Pistrang, N., Rozental, A., Egan, S., Wade, T. et al. (2019). A Randomized Controlled Trial of Guided Internet-Based Cognitive Behavioural Therapy for Perfectionism: Effects on Psychopathology and Trans-Diagnostic Processes. Journal of Behavior Therapy and Experimental Psychiatry, 64, 113-122. https://doi.org/10.1016/j.jbtep.2019.03.007

La Mela, C., Maglietta, M., Caini, S., Casu, G. P., Lucarelli, S., Mori, S., \& Ruggiero, G. M. (2015). Perfectionism, Weight and Shape Concerns, and Low Self-Esteem: Testing a Model to Predict Bulimic Symptoms. Eating Behaviors, 19, 155-158. https://doi.org/10.1016/j.eatbeh.2015.09.002

Limburg, K., Watson, H. J., Hagger, M. S., \& Egan, S. J. (2017). The Relationship between Perfectionism and Psychopathology: A Meta-Analysis. Journal of Clinical Psychology, 73, 1301-1326. https://doi.org/10.1002/jclp.22435

Linnett, R. J., \& Kibowski, F. (2020). A Multidimensional Approach to Perfectionism and Self-Compassion. Self and Identity, 19, 757-783. https://doi.org/10.1080/15298868.2019.1669695

Lipson, S. K., Lattie, E. G., \& Eisenberg, D. (2019). Increased Rates of Mental Health Service Utilization by U.S. College Students: 10-Year Population-Level Trends (2007-2017). Psychiatric Services, 70, 60-63. https://doi.org/10.1176/appi.ps.201800332

Lo, A., \& Abbott, M. J. (2019). Self-Concept Certainty in Adaptive and Maladaptive Perfectionists. Journal of Experimental Psychopathology, 10, 1-12. https://doi.org/10.1177/2043808719843455

Macedo, A., Marques, C., Quaresma, V., Soares, M. J., Amaral, A. P., Araujo, A. I., \& Pereira, A. T. (2017). Are Perfectionism Cognitions and Cognitive Emotion Regulation Strategies Mediators between Perfectionism and Psychological Distress? Personality and Individual Differences, 119, 46-51. https://doi.org/10.1016/j.paid.2017.06.032

Malinowski, A. S., Veselka, L., \& Atkinson, B. E. (2017). An Investigation of Vulnerability Factors for Depression. Personality and Individual Differences, 107, 126-130. https://doi.org/10.1016/j.paid.2016.11.049

Mehr, K. E., \& Adams, A. C. (2016). Self-Compassion as a Mediator of Maladaptive Per- 
fectionsim and Depressive Symptoms in College Students. Journal of College Student Psychotherapy, 30, 132-145. https://doi.org/10.1080/87568225.2016.1140991

Moate, R. M., Gnilka, P. B., West, E. M., \& Rice, K. G. (2019). Doctoral Student Perfectionism and Emotional Wellbeing. Measurement and Evaluation in Counseling and Development, 52, 145-155. https://doi.org/10.1080/07481756.2018.1547619

Mohammadian, Y., Mahaki, B., Dehghani, M., Vahid, M. A., \& Lavasani, F. F. (2018). Investigating the Role of Interpersonal Sensitivity, Anger, and Perfectionism in Social Anxiety. International Journal of Preventive Medicine, 9, 1-12. https://doi.org/10.4103/ijpvm.IJPVM_364_16

Moher, D., Liberati, A., Tetzlaff, J., \& Altman, D. G. (2009). Preferred Reporting Items for Systematic Reviews and Meta-Analyses: The PRISMA Reporting Statement. PLoS ONE, 6, e1000097. https://doi.org/10.1371/journal.pmed.1000097

Molnar, D. S., Sirois, F. M., Flett, G. L., Janssen, W. F., \& Hewitt, P. L. (2018). Perfectionism and Health: The Roles of Health Behaviors and Stress-Related Processes. In J. Stoeber (Ed.), The Psychology of Perfectionism: Theory, Research, and Applications (pp. 200-221). London: Routledge. https://doi.org/10.4324/9781315536255-14

Moroz, M., \& Dunkley, D. M. (2015). Self-Critical Perfectionism and Depressive Symptoms: Low Self-Esteem and Experiential Avoidance as Mediators. Personality and Individual Differences, 87, 174-179. https://doi.org/10.1016/j.paid.2015.07.044

Muyan, M., \& Chang, E. C. (2015). Perfectionism as a Predictor of Suicidal Risk in Turkish College Students: Does Loneliness Contribute to Further Risk? Cognitive Therapy and Research, 39, 776-784. https://doi.org/10.1007/s10608-015-9711-7

Newman, B. N., Strickler, J. G., O’Brien, C., Lui, T., \& Lynch, M. (2019). Deconstructing Perfectionism in College Students: Patterns of Behavior, Emotion, and Cognition. Personality and Individual Differences, 145, 106-111.

https://doi.org/10.1016/j.paid.2019.03.030

Norwin J. N. (2018). Academic Procrastination and Perfectionism among College Students. International Journal of Science and Research, 7, 33-35.

O'Connor, R. (2007). The Relations between Perfectionism and Suicidality: A Systematic Review. Suicide and Life-Threatening Behavior, 37, 698-714. https://doi.org/10.1521/suli.2007.37.6.698

Ong, C. W., Lee, E. B., Krafft, J., Terry, C. L., Barrett, T. S., Levin, M. E., \& Twohig, M. P. (2019). A Randomized Controlled Trial of Acceptance and Commitment Therapy for Clinical Perfectionism. Journal of Obsessive-Compulsive and Related Disorders, 22, Article ID: 100444. https://doi.org/10.1016/j.jocrd.2019.100444

Park, H. J., \& Jeong, D. Y. (2015). Psychological Wellbeing, Life Satisfaction, and Self-Esteem among Adaptive Perfectionists, Maladaptive Perfectionists, and Non-Perfectionists. Personality and Individual Differences, 72, 165-170. https://doi.org/10.1016/j.paid.2014.08.031

Rice, K. G., \& Preusser, K. J. (2002). The Adaptive/Maladaptive Perfectonism Scale. Measurement and Evaluation in Counseling and Development, 34, 210-222. https://doi.org/10.1080/07481756.2002.12069038

Rice, K. G., \& Richardson, C. M. E. (2014). Classification Challenges in Perfectionism. Journal of Counseling Psychology, 61, 641-648. https://doi.org/10.1037/cou0000040

Rozental, A., Shafran, R., Wade, T., Egan, S. et al. (2017). A Randomized Controlled Trial of Cognitive Behavioral Internet Based Cognitive Behavior Therapy for Perfectionism Including an Investigation of Outcome Predictors. Behavior Research and Therapy, 95, 79-86. https://doi.org/10.1016/j.brat.2017.05.015

Santos, T., Marques, C., Pereirea, A., Soares, S. C., \& Pereira Macedo, A. (2018). Family 
Systems, Offspring and Eating Disorders: Can Perfectionism Close the Gaps? International Journal of Clinical Neurosciences and Mental Health, 5, 1-8.

Sedera, D., \& Lokuge, S. (2020). Flaws in Flawlessness: Perfectionism as a New Technology Driven Mental Disorder. Forty-First International Conference on Information Systems, India.

Segrin, C., Kauer, T. B., \& Burke, T. J. (2019). Indirect Effects of Family Cohesion on Emerging Adult Perfectionism through Anxious Rearing and Social Expectations. Journal of Child and Family Studies, 28, 2280-2285. https://doi.org/10.1007/s10826-019-01444-2

Shafran, R., Coughtrey, A., \& Kothari, R. (2016). New Frontiers in the Treatment of Perfectionism. International Journal of Cognitive Therapy, 9, 156-170. https://doi.org/10.1521/ijct.2016.9.2.156

Shafran, R., Wade, T. D., Egan, S. J., Kothari, R., Allcott-Watson, H., Carlbring, P., \& Anderson, G. (2017). Is the Devil in the Details? A Randomized Controlled Trial of Guided Internet-Based CBT for Perfectionism. Behaviour Research and Therapy, 95, 99-106. https://doi.org/10.1016/j.brat.2017.05.014

Shahar, G. (2006). An Investigation of the Perfectionism/Self-Criticism Domain of the Personal Style Inventory. Cognitive Therapy and Research, 30, 185-200. https://doi.org/10.1007/s10608-006-9032-y

Shahnaz, A., Saffer, B. Y., \& Klonsky, E. D. (2018). The Relationship of Perfectionsim to Suicide Ideation and Attempts in a Large Online Sample. Personality and Individual Differences, 130, 117-121. https://doi.org/10.1016/j.paid.2018.04.002

Sharma, M. (2016). Theoretical Foundations of Health Education and Health Promotion (3rd ed.). Burlington, MA: Jones \& Bartlett.

Smith, M. M., Saklofske, D. H., Yan, G., \& Sherry, S. B. (2017). Does Perfectionism Predict Depression, Anxiety, Stress, and Life Satisfaction after Controlling for Neuroticism? Journal of Individual Differences, 38, 63-70.

https://doi.org/10.1027/1614-0001/a000223

Smith, M., Sherry, S. B., Chen, S., Saklofske, D., Muhquash, C., Flett, G., \& Hewitt, P. (2018). The Perniciousness of Perfectionism: A Meta-Analytic Review of the Perfectionism-Suicide Relationship. Journal of Personality, 86, 522-542. https://doi.org/10.1111/jopy.12333

Starley, D. (2019). Perfectionism: A Challenging but Worthwhile Research Area for Educational Psychology. Educational Psychology in Practice, 35, 121-146. https://doi.org/10.1080/02667363.2018.1539949

Stewart, M. A., \& De George-Walker, L. (2014). Self-Handicapping, Perfectionism, Locus of Control and Self-Efficacy: A Path Model. Personality and Individual Differences, 66, 160-164. https://psycnet.apa.org/doi/10.1016/j.paid.2014.03.038 https://doi.org/10.1016/j.paid.2014.03.038

Stoeber, J. (2018). Comparing Two Short Forms of the Hewitt-Flett Multidimensional Perfectionism Scale. Assessment, 25, 578-588.

https://doi.org/10.1177/1073191116659740

Stoeber, J., \& Yang, H. F. (2015). Physical Appearance Perfectionism Explains Variance in Eating Disorder Symptoms above General Perfectionism. Personality and Individual Differences, 86, 303-307. https://doi.org/10.1016/j.paid.2015.06.032

Thojampa, S., \& Sarnkhaowkhom, C. (2019). The Social Cognitive Theory with Diabetes: Discussion. International Journal of Caring Sciences, 12, 1251-1255.

Tonta, K. E., Howell, J. A., Hasking, P. A., Boyes, M. E., \& Clarke, P. J. (2018). Attention Biases in Perfectionism: Biased Disengagement of Attention from Emotionally Nega- 
tive Stimuli. Journal of Behavior Therapy and Experimental Psychiatry, 64, 72-79. https://doi.org/10.1016/j.jbtep.2019.02.009

Valentine, E. G., Bodill, K. O., Watson, H. J., Hagger, M. S., Kane, R. T., Anderson, R. A., \& Egan, S. J. (2018). A Randomized Controlled Trial of Unguided Internet Cognitive-Behavioral Treatment for Perfectionism in Individuals Who Engage in Regular Exercise. International Journal of Eating Disorders, 51, 984-988.

https://doi.org/10.1002/eat.22888

Vanstone, D. M., \& Hicks, R. E. (2019). Transitioning to University: Coping Styles as Mediators between Adaptive-Maladaptive Perfectionism and Test Anxiety. Personality and Individual Differences, 141, 68-75. https://doi.org/10.1016/j.paid.2018.12.026

Vidic, Z., \& Cherup, N. (2019). Mindfulness in Classroom: Effect of Mindfulness-Based Relaxation on College Students' Stress, Resilience, Self Efficacy and Perfectionism. College Student Journal, 53, 130-142.

Wade, T. D., O’Shea, A., \& Shafran, R. (2016). Perfectionism and Eating Disorders. In F. M. Sirois, \& D. S. Molnar (Eds.), Perfectionism, Health, and Wellbeing (pp. 205-222). Cham: Springer. https://doi.org/10.1007/978-3-319-18582-8_9

Walton, G. E., Hibbard, D. R., Couglin, C., \& Coyl-Shepherd, D. D. (2020). Parenting, Personality, and Culture as Predictors of Perfectionism. Current Psychology, 39, 681-693. https://doi.org/10.1007/s12144-018-9793-y

Wimberley, T. E., Mintz, L. B., \& Suh, H. (2016). Perfectionism and Mindfulness: Effectiveness of a Bibliotherapy Intervention. Mindfulness, 7, 433-444.

https://doi.org/10.1007/s12671-015-0460-1

Yildiz, M., Duru, H., \& Eldeleklioglu, J. (2020). Relationship between Parenting Style and Multidimensional Perfectionsim: A Meta-Analysis Study. Education Sciences: Theory and Practice, 20, 16-35.

Zetterberg, M., Carlbring, P., Anderson, G., Berg, M., Shafran, R., \& Rozental, A. (2019). Internet-Based Cognitive Behavioral Therapy of Perfectionism: Comparing Regular Therapist Support and Support upon Request. Internet Interventions, 17, Article ID: 100237. https://doi.org/10.1016/j.invent.2019.02.001 